\title{
Revascularização direta do miocárdio com as duas artérias mamárias internas: análise de 442 casos
}

\author{
Marcelo B. JATENE*, Luiz Boro PUIG*, Fábio B. JATENE*, Antônio F. RAMIRES*, Sérgio de Almeida \\ OLIVEIRA*, Luís Alberto DALLAN*, Ronaldo D. FONTES*, Adib D. JATENE*
}

RBCCV 44205-109

JATENE, M. B.; PUIG, L. B.; JATENE, F. B.; RAMIRES, A. F.; OlIVEIRA, S. A.; DALLAN, L. A.; FONTES, R. D.; JATENE, A. D. - Revascularização direta do miocárdio com as duas artérias mamárias internas: análise de 442 casos. Rev. Bras. Cir. Cardiovasc., 5(2): 71-78, 1990.

RESUMO: No periodo de julho de 1984 a novembro de 1989, foram submetidos a revascularização direta do miocárdio 4958 pacientes. Em $54 \%$ foi empregada pelo menos uma artéria mamária interna (AMI) e em $442(8,91 \%)$ pacientes as duas artérias mamárias interna direita (AMID) e interna esquerda (AMIE), associadas ou não a outros tipos de enxertos. A idade variou de 30 a 78 anos (média de 52,7 anos) e 399 pacientes eram do sexo masculino. Os pacientes que receberam as duas AMls foram classificados em 4 grupos: Grupo 1: AMIE para descendente anterior (DA) e AMID para diagonal (Di); diagonalis (DI), circunflexa $(\mathrm{Cx})$ ou seus ramos marginais em posição retro-aórtica $232(52,4 \%)$ pacientes; Grupo 2: AMIE para DA e MID para coronária direita (CD) ou seus ramos $135(30,5 \%)$ pacientes; Grupo 3: AMIE para ramos da Cx e AMID para DA (enxerto livre) ou seus ramos $48(10,8 \%)$ pacientes; Grupo 4: outras associações $27(6 \%)$ pacientes. Em todos os grupos, as revascularizaçōes foram ou nảo complementadas com pontes de veia safena, artéria epigástrica inferior, artéria gastro-epiplóica e condutos sintéticos (como Gorotex). A média de ramos coronarianos revascularizados foi de 3,17 pontes por pacientes. As principais complicaçōes imediatas observadas foram: insuficiência respiratória $37(8,3 \%)$ pacientes, baixo débito $23(5,2 \%)$ pacientes; infarto do miocárdio $16(3,6 \%)$; deiscência e infecção de esterno $15(3,6 \%)$. A mortalidade imediata global foi de $4,97 \%$ (22 pacientes) e as principais causas de óbito foram: falência de multiplos órgãos em 7 pacientes, insuficiência miocárdica em $6(27,3 \%)$. Observou-se maior índice de mortalidade em pacientes com idade superior a 60 anos e com severa disfunçāo ventricular. A melhor associaçāo enxerto/coronária foi obtida quando da anastomose da AMIE com a DA (patência de $93,8 \%$ ). O emprego de duas mamárias vem sendo incrementado no Serviço, bem como o uso de outros enxertos arteriais, especialmente a artéria epigástrica em função dos aceitáveis índices de morbi-mortalidade.

DESCRITORES: miocárdio, revascularização direta.

\section{INTRODUÇĀO}

Desde o início da cirurgia de revascularização direta do miocárdio (CRM), no final da década de $1960^{4,15}$, tem-se buscado um tipo de enxerto, quer seja arterial ou venoso, que proporcione fluxo adequado à coronária a ser revascularizada, seja pérvio por longo tempo com mínimo de complicaçōes na obtenção e implante com baixa morbidade e baixa mortalidade. No entanto, qualquer enxerto apresenta pontos favoráveis e desfavoráveis no seu manuseio e a análise crítica e criteriosa

Trabalho realizado no Instituto do Coração do Hospital das Clinicas da Faculdade de Medicina da Universidade de São Paulo. São Paulo, SP, Brasil.

Apresentado ao 17: Congresso Nacional de Cirurgia cardiaca. Belo Horizonte, MG, 6 e 7 de abril, 1990.

Laureado com o "Prêmio Nacional de Cirurgia Cardiaca - 1990".

* Do Instituto do Coração do Hospital das Clinicas da Faculdade de Medicina da Universidade de São Paulo.

Endereço para separatas: Marcelo Jatene. Av. Dr. Enéas de Carvalho Aguiar, 44. Divisão Cirúrgica. 05403. São Paulo, SP, Brasil 
JATENE, M. B.; PUIG, L. B.; JATENE, F. B.; RAMIRES, A. F.; OLIVEIRA, S. A.; DALlAN, L. A.; FONTES, R. D.; JATENE, A. D. - Revascularização direta do miocárdio com as duas artérias mamárias internas: análise de 442 casos. Rev. Bras. Cir. Cardiovasc., 5(2): 71-78, 1990.

de cada paciente é que vai direcionar a escolha do meIhor enxerto.

A partir de estudos realizados no final da década de 70 e início da década de $80^{1,4,5,8,11,13}$, que avaliaram a longo prazo os enxertos em uso, veia safena e artéria mamária interna, a maioria dos grupos passou a utilizar, com a máxima freqüência possivel, os enxertos com a artéria mamária. Isto deveu-se ao fato de que esta apresentara patência acima de $90 \%$ em 10 anos, em relação aos enxertos com veia safena, que era ao redor de $60 \%$ em oito anos.

No entanto, o que parece ser o enxerto ideal apresenta alguns pontos desfavoráveis: maior dificuldade técnica, maior índice de complicaçōes pós-operatórias, sobretudo nos pacientes em que se utilizam as duas artérias mamárias internas.

Baseados nestes e em outros aspectos, procuramos mostrar a experiência de nossa Instituição no manuseio com este grupo de pacientes, identificando aspectos que julgamos importantes para utilização deste procedimento.

\section{CASUISTICA E MÉTODOS}

No período compreendido entre junho de 1984 e novembro de 1989 , foram submetidos a CRM 4958 pacientes, no Instituto do Coraçāo da FMUSP. Destes, 442 $(8,91 \%)$ foram submetidos a CRM com utilização das duas artérias mamárias internas, isoladas ou associadas a outros enxertos. A idade variou de 30 a 78 anos (média de 52,7 anos), e $399(90,3 \%)$ pacientes eram do sexo masculino.

Trinta e sete $(8,3 \%)$ pacientes tinham idade inferior a 40 anos, $107(24,2 \%)$ idade superior a 61 anos e os demais $298(67,4 \%$ ) idade entre 41 e 60 anos (Tabela 1). Trinta $(6,8 \%)$ pacientes eram safenectomizados ou tinham inviabilidade de uso da veia safena e $32(7,2 \%)$ já haviam sido submetidos a CRM prévia. Quanto à posição anatômica dos enxertos, os pacientes foram divididos em quatro grupos, a saber: Grupo 1) 232 (52,4\%) - mamária interna esquerda (MIE) para descendente anterior (DA) e mamária interna direita (MID) para circunflexa (Cx) ou ramos marginais, diagonalis (DI) ou ramos diagonais (Di) em posição retroaórtica; Grupo 2) 135 $(30,5 \%)$ - MIE para DA e MID para coronária direita (CD) ou ramos descendente e ventricular posterior; Grupo 3) $48(10,8 \%)$ - MIE para marginal esquerda (ME) e MID para DA ou Di tipo enxerto livre; Grupo 4) 27 $(6,1 \%)$ - miscelânia com diferentes associaçōes entre as duas artérias mamárias (Tabela 2). Foram utilizados 819 enxertos in situ, sendo 440 com MIE e 379 com MID; 65 enxertos livres foram empregados, sendo 63 de MID e dois de MIE. Foram associadas 505 pontes de safena, sete enxertos de artéria epigástrica inferior, quatro pontes de veia cefálica, quatro pontes com PTFE (Goretex) e um enxerto livre de artéria gastro-epiplóica. Cinco pacientes receberam enxertos seqüenciais com as artérias mamárias. A média foi de 3,17 enxertos por paciente. As características cineangiocoronariográficas pré-operatórias estāo expostas na Tabela 3.

TABELA 1

CASUISTICA CONFORME IDADE

\begin{tabular}{lr}
\hline$<30$ anos & $1(0,2 \%)$ \\
31 a 40 anos & $36(8,1 \%)$ \\
41 a 50 anos & $121(27,4 \%)$ \\
51 a 60 anos & $177(40,0 \%)$ \\
61 a 70 anos & $98(22,2 \%)$ \\
$>70$ anos & $9(2,0 \%)$ \\
\hline
\end{tabular}

TABELA 2

LOCAL DAS ANASTOMOSES

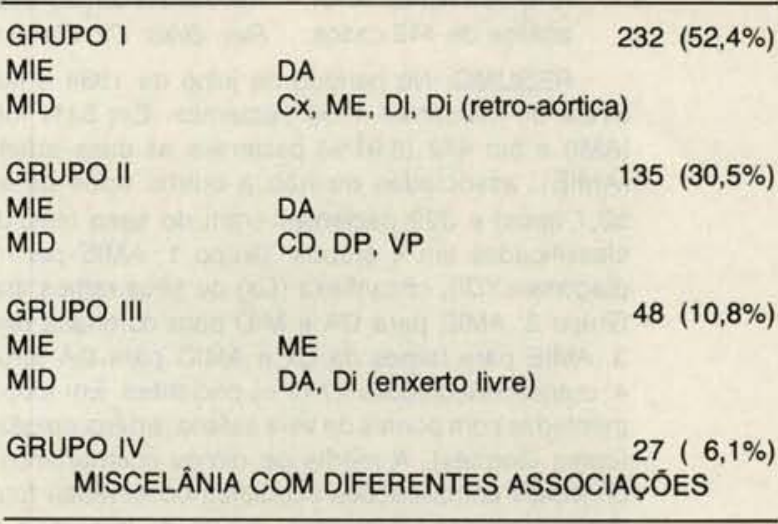

\section{TABELA 3 \\ CRITÉRIOS PARA INDICACCAOO CIRÚRGICA (CINECORONARIOGRAFIA)}

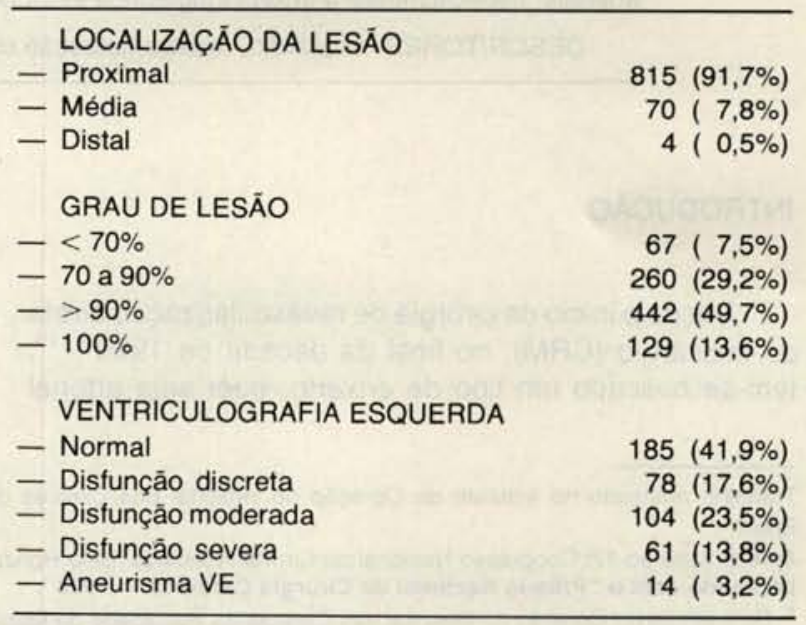

-IAM prévio em 237 pacientes 
JATENE, M. B.; PUIG, L. B.; JATENE, F. B.; RAMIRES, A. F.; OLIVEIRA, S. A.; DALLAN, L. A.; FONTES, R. D.; JATENE, A. D. - Revascularização direta do miocárdio com as duas artérias mamárias internas: análise de 442 casos. Rev. Bras. Cir. Cardiovasc., 5(2): 71-78, 1990.

Quanto à técnica cirúrgica empregada na grande maioria dos pacientes, após toracotomia médio-esternal, foi realizada a abertura das pleuras durante a dissecção das artérias mamárias com posterior drenagem pleural bilateral, além de dreno mediastinal. A proteção miocárdica utilizada foi com hipotermia moderada $\left(32^{\circ} \mathrm{C}\right)$ associada a clampeamento intermitente da aorta em 374 $(84,6 \%)$ pacientes e em $68(15,4 \%)$ foi empregada hipotermia moderada $\left(28^{\circ} \mathrm{C}\right)$ associada a cardioplegia cristalóide anterógrada. Quanto aos procedimentos associados, em 10 pacientes foi realizada, além da CRM, aneurismectomia de ventrículo esquerdo (VE); em um, correção de aneurisma de aorta ascendente; em um, endarterectomia de carótida esquerda e em um, correção de hérnia incisional da porção inferior da incisão torácica pregressa (caso de reoperação). A indicação cirúrgica foi eletiva em 417 (94,3\%) e, nos outros 25 (5,7\%), foi em situação de urgência, caracterizada por angina incontrolável com alteração eletrocardiográfica, sem instabilidade hemodinâmica severa ou choque cardiogênico.

\section{RESULTADOS}

A mortalidade hospitalar foi de 4,97\% (22 pacientes) e as principais causas de óbito foram: falência de múltiplos órgãos em sete $(31,8 \%)$; insuficiência miocárdica em seis $(27,3 \%)$; insuficiência respiratória (IR) em quatro $(18,3 \%)$; infarto agudo do miocárdio (IAM) em dois $(9,1 \%)$; hemorragia digestiva alta em um $(4,5 \%)$; anóxia cerebral em um $(4,5 \%)$ e coagulopatia em um $(4,5 \%)$. Dois dos pacientes com falência de múltiplos órgão tiveram infarto agudo do intra-operatório.

Não houve óbito em pacientes com idade inferior a 50 anos, num total de 158 . Observaram-se sete $(3,95 \%)$ óbitos, num total de 177 pacientes com idades entre 51 e 60 anos; 11 (11,22\%) óbitos em 98 pacientes entre 61 e 70 anos; e quatro $(44,44 \%)$ óbitos em nove pacientes com idade superior a 70 anos. Do ponto de vista estatístico ${ }^{6}$, o valor do $\times 2=46,39$ e $p<0,0001$ evidencia uma diferença significante entre a proporção total de óbito dos quatro grupos. Desta forma, prosseguiu-se ao teste confrontando o grupo com a faixa etária acima dos 70 anos com os demais, obtendo-se $\times 2=$ 30,26 , que é significante. Fez-se, em seguida, a comparação dos três outros grupos e obteve-se $\times 2=16,13$ (significante) ${ }^{6}$.

A partição desse valor de $\times 2$ mostrou diferença significante entre o grupo com faixa etária de 61 a 70 a e os grupos com idade inferior a 50 anos e entre 51 e 60 anos, que, por sua vez, são iguais. Estes dados estāo representados na Tabela 4.

Com relação à função ventricular no pré-operatório, foram observados quatro $(2,16 \%)$ óbitos entre 185 pacientes com função ventricular normal; quatro $(5,13 \%)$ óbitos entre 78 pacientes com discreta disfunçāo; cinco
$(4,81 \%)$ em 104 pacientes com disfunção moderada; sete $(11,48 \%)$ óbitos num total de 61 pacientes com severa disfunção ventricular e dois $(14,29 \%)$ óbitos em 14 pacientes com aneurisma de VE. O teste estatístico mostrou $\times 2$ total $=11,12$ com $p=0,025$, que evidenciou uma diferença significante entre as proporçōes de óbito dos cinco grupos analisados. Dando seqüência ao teste, evidenciou-se que, entre os três grupos (funçāo de VE normal, discreta disfunçāo e disfunção moderada), não houve diferença significante, bem como entre os grupos com severa disfunção e aneurisma de VE; porém, quando se comparam os três primeiros grupos com os dois últimos, observa-se diferença significante $(x 2=9,40$ e p $=0,025$ ). Estes dados estão na Tabela 5 .

Quanto ao sexo, observaram-se $17(4,26 \%)$ óbitos entre os 399 pacientes do sexo masculino e cinco $(11,63 \%)$ óbitos entre os 43 pacientes do sexo feminino. $\mathrm{O}$ teste estatístico mostrou $\times 2=4,45$ e $p=0,035$, que evidenciou diferença significante entre as proporçōes de óbito, comparando-se os dois grupos.

Com relaçāo ao caráter da indicação cirúrgica, houve $21(5,0 \%)$ óbitos entre 417 pacientes operados eletiva-

TABELA 4

MORTALIDADE X IDADE

\begin{tabular}{|c|c|c|c|c|c|}
\hline Faixa etária & $\begin{array}{l}\text { Nide } \\
\text { Casos }\end{array}$ & $\begin{array}{l}\text { Mo } \\
N:\end{array}$ & $\begin{array}{r}\text { dade } \\
\%\end{array}$ & & $\begin{array}{c}\text { Estatistica } \\
\times 2\end{array}$ \\
\hline$<=50$ & 158 & 0 & 0,00 & & \\
\hline $51->60$ & 177 & 7 & 3,95 & $\begin{array}{l}16.13 \\
\text { (SIG) }\end{array}$ & $\begin{array}{c}\text { (NS) } \\
13,38 \\
\text { (SIG) }\end{array}$ \\
\hline $61->70$ & 98 & 11 & 11,22 & $\begin{array}{l}30,26 \\
\text { (SIG) }\end{array}$ & \\
\hline$>70$ & 9 & 4 & 44.44 & & \\
\hline
\end{tabular}

$\mathrm{X} 2$ Total $=46,30 \mathrm{P}<0,0001$

TABELA 5

MORTALIDADE X FUNÇÃO VENTRICULAR

\begin{tabular}{|c|c|c|c|c|}
\hline Função & N: de & & ade & Estatistica \\
\hline Ventricular & Casos & $N:$ & $\%$ & $x 2$ \\
\hline Normal & 185 & 4 & 2,16 & \\
\hline Disf. discreta & 78 & 4 & 5,13 & $\begin{array}{l}1,52 \\
\text { (NS) }\end{array}$ \\
\hline Disf. moderada & 104 & 5 & 4,81 & $\begin{array}{l}9,40 \\
\text { (NS) }\end{array}$ \\
\hline Disf. severa & 61 & 7 & 11,48 & $\begin{array}{l}0,20 \\
\text { (NS) }\end{array}$ \\
\hline
\end{tabular}

$\mathrm{X} 2$ Total $=11,12 \mathrm{P}=0,025$ 
JATENE, M. B.; PUIG, L. B.; JATENE, F. B.; RAMiRES, A. F.; OliVeiRA, S. A.; DALlan, L. A.; FOnTES, R. D.; JATENE, A. D. - Revascularização direta do miocárdio com as duas artérias mamárias internas: análise de 442 casos. Rev. Bras. Cir. Cardiovasc., 5(2): 71-78, 1990.

mente e um $(4,0)$ óbito nos 25 pacientes operados em caráter de urgência. A análise estatística mostrou $\times 2$ $=0,054$ e $p=0,817$ (NS), que não evidencia diferença significante entre as proporções de óbito em um grupo e outro. Com relação ao tipo de proteção miocárdica utilizada, observados $15(4,0 \%)$ óbitos entre os 374 pacientes operados com pinçamento intermitente e sete $(10,3 \%)$ óbitos entre os 68 pacientes operados com cardioplegia. A análise estatística mostrou $\times 2=0,054 \mathrm{e}$ $p=0,817$ (NS) que evidencia diferença significante entre as proporçōes de óbito em um grupo e outro.

Quanto às complicações pós-operatórias, procurouse dividi-las em complicaçōes específicas ao procedimento, que guardaram relaçāo mais direta com o ato operatório, e outras inespecíicas. Dentre as complicaçōes específicas, as que julgamos mais importantes foram: infarto agudo do miocárdio (IAM) em 16 (3,6\%) pacientes, que guardaram ou não relação com a área miocárdica tratada pelas artérias mamárias, como veremos a seguir. Observamos seis $(1,3 \%)$ pacientes com sangramento pós-operatório que necessitaram reoperação imediata, sendo que, em quatro pacientes, foram identificadas causas cirúrgicas e, em outros dois, apenas coagulopatia; em $15(3,3 \%)$ pacientes ocorreu infecção esternal com mediastinite, sendo que, em nove $(2,0 \%)$, o tratamento foi clínico, além de curativos diários e, em seis $(1,3 \%)$, houve necessidade de reoperação, com limpeza mediastinal e ressutura de esterno; todos tiveram boa evolução pós-operatória, com exceção de um paciente que, após resolução do quadro infeccioso, apresentou episódio de hemorragia digestiva alta importante, evoluindo para óbito, no 40: dia de pós-operatório.

Quanto às complicações inespecíficas, as mais comumente observadas foram as respiratórias em 37 $(8,3 \%)$, onde caracterizamos como IR quadros onde 0 paciente necessitou suporte ventilatório mecânico por período superior a 48 horas, ou hipoxemia com Po2 < $50 \mathrm{mmHg}$ ou em casos de reintubaçāo por falência muscular; foi realizada traqueostomia em quatro pacientes. Dos 37 pacientes com IR, houve evolução fatal em quatro, sendo que os demais, em maior ou menor tempo, tiveram boa evolução. Em $23(5,2 \%)$ pacientes houve evolução com síndrome de baixo débito, caracterizadas, nos casos mais graves, por choque cardiogênico e, nos casos mais leves, por hipotensão discreta em uso de drogas vaso-ativas; em oito pacientes o baixo débito teve relação com IAM pós-operatório e oito pacientes evoluíram para óbito. A grande maioria destes pacientes recebeu suporte circulatório com balão intra-aórtico (BIA), bem como controle hemodinâmico através de cateter de Swan-Ganz. As demais complicaçōes pós-operatórias, específicas ou inespecíficas, estâo expostas na Tabela 6.

Analisando especificamente os 16 pacientes que apresentaram IAM no pós-operatório, notamos que dois apresentaram IAM na área revascularizada pela MIE
TABELA 6

COMPLICAÇÓES POSS-OPERATÓRIAS ESPECIFICAS AO PROCEDIMENTO

\begin{tabular}{lr}
\hline Enfisema subcutâneo & $42(9,5 \%)$ \\
Atelectasias pulmonares & $25(5,6 \%)$ \\
Derrame pleural unilateral & $22(4,9 \%)$ \\
Derrame pleural bilateral & $20(4,5 \%)$ \\
Infarto agudo do miocárdio & $16(3,6 \%)$ \\
Sangramento por coagulopatia & $12(2,7 \%)$ \\
Sangramento pós-operatório - reoperados & $6(1,3 \%)$ \\
Infecção de esterno - não reoperados & $9(2,0 \%)$ \\
Infecção de esterno - reoperados & $6(1,3 \%)$ \\
\hline
\end{tabular}

INESPECIFICAS

Insuficiência respiratória

$37(8,3 \%)$

Sindrome de baixo débito

$23(5,2 \%)$

Distensão abdominal

$21(4,7 \%)$

Arritmias

Insuficiência renal aguda

$18(4,1 \%)$

$13(2,9 \%)$

Acidente vascular cerebral

(sendo um caso para DA e um para ME in situ), com boa evolução; em sete pacientes o IAM correnpondeu à área revascularizada pela MID (quatro para $C D$, dois para ME e um para DA, enxerto livre), com evolução fatal em três pacientes; em três casos houve relação do IAM com a área da ponte de veia safena, com boa evolução; quatro pacientes apresentaram IAM em território de artérias coronárias revascularizadas, com um óbito neste grupo. Portanto, nos 16 pacientes com IAM no pós-operatório, quatro $(25 \%)$ apresentaram evolução fatal em decorrência do IAM e os 12 (75\%) restantes tiveram boa evolução.

Nāo se observou diferença significativa de IAM em pacientes operados com pinçamento intermitente e com cardioplegia, respectivamente $12(3,2 \%)$ IAM em 374 pacientes e quatro $(5,8 \%)$ IAM em 68 pacientes $(\times 2=$ 1,18 e $p=0,278$ NS).

No que diz respeito à evolução tardia, observaramse três $(0,6 \%)$ óbitos tardios, dois de causa cardiaca (um por IAM, 48 meses após e um por insuficiência miocárdia, 60 meses após) e um por causa não cardiaca (pancreatite aguda, 58 meses após).

Foram realizados reestudos hemodinâmicos em 32 pacientes em diferentes fases de pós-operatório; 10 pacientes foram reestudados na fase hospitalar, como controle imediato do procedimento, sendo que apenas um paciente apresentava aclusão precoce em uma das mamárias (MID); os 22 pacientes restantes foram reestudados por presença de sintomatologia, em períodos que variaram de dois meses a cinco anos de pós-operatório. Nesse grupo, 13 pacientes tinham as duas mamárias pérvias; sete pacientes apresentaram oclusão da MID e dois pacientes as duas mamárias ocluidas. Quanto 
JATENE, M. B.; PUIG, L. B.; JATENE, F. B.; RAMIRES, A. F.; OLIVEIRA, S. A.; DALLAN, L. A.; FONTES, R. D.; JATENE, A. D. - Revascularização direta do miocárdio com as duas artérias mamárias internas: análise de 442 casos. Rev. Bras. Cir. Cardiovasc., 5(2): 71-78, 1990.

à posição da ąrtéria mamária e a coronária tratada, observou-se que as 32 MIE eram in situ, sendo 22 para DA, com uma $(4,5 \%)$ ocluida; quatro para DI e seis para Cx, com uma $(16,6 \%)$ ocluída, num total de duas $(6,2 \%)$ MIE ocluídas em 3 reestudadas. Quanto à MID, 29 eram in situ, sendo 13 para $\mathrm{Cx}$ em posiçāo retro-arórtica, com duas $(15,3 \%)$ ocluídas; nove para CD com seis $(66,6 \%)$ ocluídas, seis para $D$; em posição retro-aórtica com uma $(16,6 \%)$ ocluida e uma para DA, num total de 29 MID in situ com nove $(31,0 \%)$ ocluidas; três MID foram implantadas na DA, como enxerto livre com uma $(33,3 \%)$ ocluida. No total das MID, observaram-se $10(31,2 \%)$ artérias ocluídas em 32 reestudadas. A patência da MIE foi de $93,8 \%$ contra $68,7 \%$ da MID. O pequeno número de casos nāo favoreceu estudo estatístico mais apurado desses resultados.

\section{DISCUSSÃO}

A revascularização direta do miocárdio com as duas artérias mamárias internas vem recebendo, com o passar dos anos, a adesão cada vez maior de grupos cirúrgicos, à medida em que os resultados a longo prazo vão demonstrando os melhores resultados do enxerto de mamária, em comparação com outros tipos de enxerto. $\mathrm{Na}$ nossa experiência com estes 442 pacientes, em 1984, apenas $5,3 \%$ dos pacientes revascularizados recebiam as duas artérias mamárias, contra $15,5 \%$ em 1989, com aumento progressivo com o passar dos anos. Quanto à patência do enxerto, em nosso material, $93,8 \%$ das MIE se mostraram pérvias, em período de reestudo de dois a 60 meses; a MID apresentou, no mesmo período, patência de $68,7 \%$, em 32 pacientes reestudados. TECTOR et alii ${ }^{15}$ mostraram patência ao redor de $94 \%$ da MIE, em periodo de seguimento de 60 a 108 meses. GREEN et alii ${ }^{7}$ demonstraram patência de $92 \%$ das artérias mamárias, em cinco anos, contra $83 \%$ para veia safena. GRONDIN et alii ${ }^{8}$ demonstraram patência das mamárias internas em 10 anos de $84,1 \%$ contra $52,8 \%$ da veia safena. BARNER ${ }^{1}$ mostrou que, em um ano, $100 \%$ das MIE estavam patentes, bem como $96 \%$ das MID. LYTLE et alii " demonstraram que, em período superior a cinco anos, a patência dos enxertos de mamária era ao redor de $90 \%$ e citou que a MIE para DA mostrou patência de $96 \%$, no mesmo período. OKIES et alii ${ }^{12}$ demonstraram, em cinco anos, patência da MIE para DA de $81 \%$ contra $64 \%$ de veia safena para DA e, em 10 anos, respectivamente, $69 \%$ e $45 \%$. SINGH et alii ${ }^{14}$ demonstraram em média de 6,8 anos patência dos enxertos de mamária de $94 \%$ contra $30 \%$ de veia safena. BARNER et alii ${ }^{2}$ demonstraram patência da MIE de $96,4 \%$ em um ano, $88,1 \%$ em cinco a 10 anos; a MID mostrou patência de $92,8 \%$ em um ano e $84,6 \%$ em cinco anos. LYTLE et alii ${ }^{10}$ demonstraram patência da MIE em um periodo médio de $26 \mathrm{~m}$ e $82 \%$ da MID. Em nosso material, a melhor associação enxerto-coronária foi da MIE para DA, com 22 enxertos reestudados, com apenas um (4,5\%) ocluído. A associaçāo que obteve pior resultado foi da MID para CD; seis $(66,6 \%)$ enxertos ocluídos em nove reestudados.

Quanto à mortalidade hospitalar, há resultados que diferem significativamente na literatura, como, por exemplo: LYTLE et alii ${ }^{10}$ não obtiveram óbito hospitalar em uma série de 76 pacientes que receberam somente as duas artérias mamárias, ao passo que BARNER et alii ${ }^{2}$ demonstraram mortalidade de $8 \%$ quando se utilizaram de dupla mamária em 103 pacientes, contra mortalidade de $1,4 \%$ quando fizeram uso de apenas uma artéria mamária.

LITLE et alii ${ }^{9}$, em uma série de 500 pacientes que receberam dupla mamária, obtiveram taxa de mortalidade de 1,4\%. Em nosso material, a mortalidade foi de $4,97 \%$ (22 pacientes); dentre os fatores que podem influenciar no resultado imediato, podemos citar a função ventricular pré-operatória como um dos principais. TECTOR et alii ${ }^{15}$ mostraram mortalidade de $5,9 \%$ em pacientes com função de VE normal, 9,4\% com disfunção moderada e $14,3 \%$ em pacientes com severa disfunção, o que vem de encontro ao resultado obtido em nossa casuística, que demonstra mortalidade de $2,16 \%$ em pacientes com função de VE normal, $4,81 \%$ em pacientes com disfunção moderada, $11,48 \%$ com disfunção severa e $14,29 \%$ em pacientes com aneurisma de VE. Estes dados demonstram que a indicação de dupla mamária em pacientes com severa disfunção de VE deve ser muito criteriosa, levando em conta estes resultados. Em nosso material, a idade e o sexo também foram fatores importantes com relação à mortalidade. Pacientes com idade inferior a 50 anos não apresentaram óbito; com idade entre 51 e 60 anos tiveram sete $(3,95 \%)$ óbitos; entre 61 e 70 anos $11(11,22 \%)$ óbitos e quatro $(44,4 \%)$ em pacientes com idade superior a 70 anos. Houve cinco $(11,6 \%)$ óbitos em mulheres e $17(4,2 \%)$ em homens. LYTLE et alii ${ }^{9}$ demonstraram que idade é variável constante e associada com aumento da morbi-mortalidade.

Com relaçāo às complicaçōes mais freqüentemente observadas, o infarto intra-operatório ocorreu em 16 $(3,6 \%)$ casos, sendo que quatro evoluíram para óbito, sendo dois em decorrência direta do IAM e dois por falência de múltiplos órgãos. LYTLE et alii ${ }^{9}$ demostratam IAM intra-operatório em períodos distintos, sendo o primeiro há mais de oito anos e o segundo mais recentemente. Os mesmos autores, em estudo de pacientes que receberam só dupla mamária, observaram $7,9 \%$ de IAM intra-operatório ${ }^{10}$.

A infecção esternal, que poderia ser esperada em um contingente maior de pacientes, devido a desvitalização do esterno, pela dissecção das duas mamárias, foi observada no nosso material em $15(3,3 \%)$ pacientes. Isto corresponde a cerca de duas vezes o indice habitual de infecção esternal global na nossa Instituição; todos tiveram boa evolução. Dados da literatura mostram BAR- 
JATENE, M. B.; PUIG, L. B.; JATENE, F. B.; RAMIRES, A. F.; OlIVEIRA, S. A.; DALLAN, L. A.; FONTES, R. D.; JATENE, A. D. - Revascularizaçāo direta do miocárdio com as duas artérias mamárias internas: análise de 442 casos. Rev. Bras. Cir. Cardiovasc., 5(2): 71-78, 1990.

NER ${ }^{1}$ com $4 \%$ de mediastinites com um óbito por IR e LYTLE et alii ${ }^{9,10}$ mostraram, em dois grupos distintos, a média de $2,8 \%$ de mediastinite pós-operatória em doentes que receberam dupla mamária.

Por fim, com relação ao sangramento no POI, observamos seis $(1,3 \%)$ pacientes que necessitaram reoperação, o que não excede à média do restante dos pacientes do nosso Serviço. Isto se deveu a uma cuidadosa revisão da hemostasia, ligadura individualizada de todos os ramos das artérias mamárias e atençāo especial à grande área de descolamento mediastinal. BARNER ${ }^{1}$ obteve $12 \%$ de sangramento no POI e COSGROVE et alii ${ }^{3}$ obtiveram $2,1 \%$ na sua casuística, sendo que LYTLE et alii ${ }^{9,}{ }^{10}$ observaram valores de $8,9 \%$ e $11,8 \%$ em dois grupos distintos.

Como conclusâo final, observamos taxa de mortalidade maior em pacientes com idade avançada ( $p<$ 0,0001 ), notadamente naqueles com idade superior a
70 anos. Em pacientes com severa disfunção ventricular ou aneurisma de VE, houve importante acréscimo da mortalidade ( $p=0,025)$. Em mulheres, se observou maior mortalidade ( $p=0,035)$ e não houve diferença quanto ao caráter da indicaçāo (eletiva ou urgência com $p=0,817$ ).

O número de IAM no intra-operatório foi aceitável e mostrou relação mais direta com a MID. A MIE mostrou melhores resultados a longo prazo (em especial para a DA), em comparaçāo com a MID, com resultados piores notadamente se anastomisada à $C D$. A taxa de sangramento foi pequena e o número de mediastinites no pósoperatório foi aceitável, correspondendo a 1,5 a duas vezes a taxa habitual para outros tipos de cirurgia.

Acreditamos que a escolha do melhor tipo de enxerto a ser utilizado em CRM deve obedecer a criteriosa seleção do paciente, levando-se todos estes fatores em consideração para que se possa obter sucesso, com o mínimo risco de complicaçōes.

RBCCV 44205-109

JATENE, M. B.; PUIG, L. B.; JATENE, F. B.; RAMIRES, A. F.; OLIVEIRA, S. A.; DALLAN, L. A.; FONTES, R. D.; JATENE, A. D. - Revascularization with double internal mammary artery: analysis of 442 patients. Rev. Bras. Cir. Cardiovasc., 5(2): 71-78, 1990.

ABSTRACT: From June 1984 to November 1989, 4958 patients (pts) were submitted to myocardial revascularization in the Heart Institute, and in $54 \%$ at least one internal mammary artery was used. In $442(8,9 \%)$ pts both intenal mammary arteries, right (RIMA) and left (LIMA) were used, isolated or associated to ther grafts. The age ranged from 30 to 78 years old $(52,7 y)$ and 399 were male. The pts were separated in 4 groups, being: G I $232(52,4 \%)$ pts - LIMA to left anterior descending artery (LAD) and RIMA to LAD branches or circumflex (CX) branches in retroaortic position; G II $135(30,5 \%)$ pts - LIMA to LAD and RIMA to right coronary artery (RCA); G III $48(10,8 \%)$ pts - LIMA to Cx branches and RIMA to LAD as a free graft; G IV $27(6,1 \%)$ pts - different associations with both arteries. The LIMA was used in situ in 440 pts and as free graft in 2; the RIMA was used in situ in 379 pts and as free graft in 63 ; The average number of grafts/pts was 3,17 , considering the association with saphenous vein and other grafts. Respiratory insufficiency and low cardiac output syndrome were the main complications in $37(8,3 \%)$ and $23(5,2 \%)$ pts, respectively; $16(3,6 \%)$ pts had post operatory myocardial infarction and $15(3,3 \%)$ had wound closure complications. The mortality rate was $4,91 \%$ ( 22 pts) and the main causes of deaths were multiple system organ failure in $7(31,8 \%)$ and myocardial insufficiency in $6(27,3 \%)$. The mortality rate was higher in pts with severe myocardial dysfunction and older than 60 years old and the best graft patency was observed when the LIMA was anastomosed to the LAD $(93,8 \%)$. The use of both intenal mammary arteries showed good results and acceptable morbidity and mortality.

DESCRIPTORS: myocardial revascularization, direct.

\section{REFERÊNCIAS BIBLIOGRÁFICAS}

1 BARNER, H. H. - Double internal mammary artery bypass. Arch. Surg., 109: 627-630, 1974.

2 BARNER, H. B.; STANDEVEN, J. W.; REESE, J. - Twelve-year experience with internal mammary artery for coronary artery bypass. J. Thorac. Cardiovasc. Surg., 90: 668-675, 1985.

3 COSGROVE, D. M.; LOO, F. D.; LYTLE, B. W.; GOORMASTIC, M.; STEWART, R. W.; GILL, C. C.; GOLDING, L. R. - Does mammary artery grafting increase surgical risk? Circulation, 72 (Supl. 2): 170-174, 1985. 
JATENE, M. B.; PUIG, L. B.; JATENE, F. B.; RAMIRES, A. F.; OlIVEIRA, S. A.; DALLAN, L. A.; FONTES, R. D.; JATENE, A. D. - Revascularizaçāo direta do miocárdio com as duas artérias mamárias internas: análise de 442 casos. Rev. Bras. Cir. Cardiovasc., 5(2): 71-78, 1990.

4 CUSHING, W. J.; MAGOVEN, G. J.; OLEARCHYK, A. S. - Internal mammary artery graft: retrospective report with 17 years survival. J. Thorac. Cardiovasc. Surg., 92: 963-964, 1986.

5 EUROPEAN CORONARY SURGERY STUDY GROUP Long-term results of prospective randomised study of coronary artery bypass surgery in stable angina pectoris. Lancet, 2: 1173-1180, 1982.

6 FLEISS, J. L. - Statistical methods for rates and proportions. 2. ed. New York, John Willy and Sons, 1981.

7 GREEN, G. E.; KEM, H. G.; ALAM, S. E.; PIERSON Jr., M. R. N.; FRIEDMAN, M. I.; DAVID, I. - Coronary bypass surgery: five-year follow-up of consectuve series of 140 patients. J. Thorac. Cardiovasc. Surg. 77: $48-56,1979$

8 GRONDIN, C. M.; CAMPEAU, L.; LESPERANCE, J.; ENJALBERT, M.; BOURASSA, M. G. - Comparison of late changes in internal mammary artery and saphenous vein grafts in two consecutive series of patients 10 years after operation. Circulation, 70 (Supl. 1) 208-212, 1984.

9 LYTLE, B. W.; COSGROVE, D. M.; LOOP, F. D.; BORSH, J.; GOORMASTIC, M.; TAYLOR, P. C. - Perioperative risk of bilateral internal mammary artery grafting: analysis of 500 cases from 1971 to 1984 . Circulation, 74 (Supl. 3): 37-41, 1986.

10 LYTLE, B. W.; COSGROVE, D. M.; SALTUS, G. L.; TAYLOR, P. C.; LOOP, F. D. - Multivessel coronary revascularization without saphenous vein: long-term results of bilateral internal mammary artery grafting. Ann. Thorac. Surg., 36: 540-547, 1983.

11 LYTLE, B. W.; LOOP, F. D.; COSGROVE, D. M.; RATLIFF N. B.; EASLEY, K.; TAYLOR, P. C. - Long-term (5 to 12 years) serial studies of internal mammary artery and saphenous vein coronary bypass grafts. J. Thorac. Cardiovasc. Surg., 89: 148-258, 1985.

12 OKIES, J. E.; PAGE, V. S.; BIGELOW, J. C.; KRAUSE, A. T. T.; SALOMON, N. W. - The left internal mammary artery: the graft of choice. Circulation, 70 (Supl. 1) 213-221, 1984.

13 SHAPIRA, N. \& LEMOLE, G. M. - Bilateral sequential IMA grafting. Ann. Thorac. Surg., 38:301, 1984. (Carta ao Editor).

14 SINGH, R. N.; SOSA, J. A.; GREEN, G. E. - Long-term fate of the internal mammary artery and saphenous vein grafts. J. Thorac. Cardiovasc. Surg., 86: 359-363, 1983.

TECTOR, A. J.; SCHMAHL, R. M.; JANSON, B.; KALLIES J. R. - The internal mammary artery graft. JAMA, 246: $2181-2183,1981$

\section{Discussão}

DR. MARCOS F. BERLINCK São Paulo, SP

Meus cumprimentos ao Dr. Marcelo, pelo trabalho muito bem apresentado, reportando uma casuística com excelentes resultados. A conduta, em nosso Serviço, é semelhante àquela aqui relatada. Com relação à técnica operatória, creio haver total concordância. Com os novos bisturís elétricos, a dissecção da artéria mamária tornou-se um procedimento simples, fácil de ser realizado e acessivel aos residentes que hoje, precocemente, o realizam. Nós temos operado 3790 pacientes com enxertos de artéria mamária, dos quais $374 \mathrm{com}$ as duas artérias mamárias. A mortalidade imediata foi agualmente baixa $(1,8 \%)$. Temos empregado a artéria mamária direita muito freqüentemente (135/374) como enxerto livre, evitando usá-la in situ em condiçōes não ideais, isto é, em segmentos mais distais, mais finos ou situações em que o pedículo fique um pouco tenso. Com o enxerto livre, podemos usar ambas as artérias mamárias em condições ideais de calibre e tensão. Assim, preferimos usar a mamária direita in situ para a artéria coronária direita, ou para revascularizar os ramos da artéria circunflexa. Quando isto não é viável, preferimos usá-la como enxerto livre, sendo, então, anastomosada à artéria descendente anterior utilizando a mamária esquerda in situ para a circunflexa. Podemos, deste modo, decidir a melhor posição para as duas mamárias. Gostaria de perguntar se o Dr. Marcelo tem alguma restrição a usar a mamária direita para a artéria coronária direita e, ainda, nos casos de reoperação, se tem preocupação de trocar uma ponte antiga funcionante para artéria com obstrução crítica por um enxerto de mamária. Concluindo e reforçando o que foi aqui demonstrado pelo Dr. Marcelo, afirmamos que, se, no passado, o uso de ambas as artérias mamárias ficava reservado para casos com falta de opção, ou para pacientes que apresentassem veias inadequadas, hoje, a tendência é de usá-las cada vez mais, pois são consideradas como enxerto ideal para revascularização mamária.

\section{DE. MARCELO JATENE (Encerrando)}

Meus agradecimentos ao Dr. Marcos, pelos comentários objetivos e pertinentes, e meus cumprimentos a ele e todo seu Serviço, sob a chefia do Prof. Sérgio de Oliveira, pela excelente casuística, com um grande número de casos e baixa taxa de mortalidade imediata. Referindo-me diretamente aos seus comentários, somos de total acordo em que, uma vez tendo sido feita a escoIha prévia das duas artérias mamárias como enxertos de eleição num determinado caso, o seu emprego ou in situ ou como enxerto livre, vai depender das circunstâncias no ato operatório, como, por exemplo, anasto- 
JATENE, M. B.; PUIG, L. B.; JATENE, F. B.; RAMIRES, A. F.; OlIVEIRA, S. A.; DALlAN, L. A.; FONTES, R. D.; JATENE, A. D. - Revascularização direta do miocárdio com as duas artérias mamárias internas: análise de 442 casos. Rev. Bras. Cir. Cardiovasc., 5(2): 71-78, 1990.

moses em seguimentos mais distais das coronárias onde a mamária utilizada in situ não tenha comprimento suficiente ou fique sob tensão; nestes casos, as utilizamos como enxerto livre. Em nosso material, 99\% das mamárias esquerdas foram utilizadas in situ, preferencialmente para a descendente anterior. Quanto à mamária direita, em $88 \%$ dos casos ela foi usada in situ, preferencialmente para a circunflexa ou seus ramos marginais em posiçāo retro-aórtica, o que acreditamos ser uma boa opção, seguindo o que foi descrito pelo Prof. Puig. Quando nos vemos frente a alguma das situações de dificuldade técnica já comentadas, as utilizamos como enxerto livre, mais comumente para a descendente anterior. A opção vai ser particular em cada caso. Quanto ao uso Ja mamária direita para a coronária direita, as restrições ao seu uso serão as mesmas já comentadas previamente, como dificuldade técnica, tensão no enxerto, anastomose distal, etc. No entanto, em nossa experiência, os resultados menos favoráveis a longo prazo, quanto à patência dos enxertos, foram obtidos quando se usou a mamária direita para a coronária direita. O número de reestudos, em nosso material, que comprovaram tal fato ainda é pequeno para que possamos concluir ou afirmar que esta associaçāo mamária coronária deve ser proscrita; no entanto, em função dos resultados obtidos, a utilização da mamária direita para a coronária direita tem sido reservada a situaçōes mais favoráveis, como em pacientes jovens, biarteriais com boa função ventricular e lesōes proximais com leito distal adequado. Quanto à troca da ponte por mamária nos casos de reoperação, alguns aspectos devem ser ressaltados: a importância da artéria em questão; a qualidade e o calibre da mamária, que, se for de pequeno calibre e a coronária de grande importância, a troca de uma antiga ponte por uma mamária menos calibrosa pode ser prejudicial quanto ao menor fluxo da mamária, se comparado ao fluxo da safena e, por fim, a qualidade cineangiográfica e intraoperatória da veia safena, que, se estiver em condições boas, não deve ser substituida. Mais uma vez, agradeço os comentários do Dr. Marcos, bem como a oportunidade de apresentar este trabalho, e reforço a importância do uso cada vez mais freqüente dos enxertos de mamária, cujos resultados a curto e longo prazo têm se mostrado bastante satisfatórios. Muito obrigado. 